\title{
Endovascular Treatment of Spontaneous and Isolated Infrarenal Acute Aortic Syndrome with Unibody Aortic Stent-Grafts
}

\author{
Felice Pecoraro $^{1,2}$ (D) $\cdot$ Ettore Dinoto $^{2} \cdot$ Domenico $^{\text {Mirabella }}{ }^{2} \cdot$ Francesca Ferlito $^{1} \cdot$ Arduino Farina $^{1}$. \\ David Pakeliani $^{3} \cdot$ Mario Lachat $^{4} \cdot$ Francesca Urso $^{1} \cdot$ Guido Bajardi $^{1}$
}

\begin{abstract}
Introduction Spontaneous acute aortic syndrome (IAAS) is rarely localized in the infrarenal aorta. The endovascular approach is preferred over conventional open surgery with fewer complications. However, dedicated endovascular devices for IAAS treatment are unavailable. The aim was to report a large single-center experience using unibody stent-grafts to address IAAS.

Methods From April 2016 to March 2019, a retrospective analysis of patients presenting spontaneous and isolated IAAS was performed. Patients addressed with the unibody stent-graft (AFX endovascular AAA system; Endologix Inc., Irvine, CA) were included in the study. Indications to IAAS treatment were persistent symptoms and/or dilated abdominal aorta $(>3 \mathrm{~cm})$. The measured outcomes were technical success; early outcomes ( $<30$ days) including mortality, morbidity, symptoms recurrence, and endoleak occurrence; and late outcomes ( $>30$ days) including mortality, symptoms recurrence, endoleak occurrence, stent-graft patency, and survival. Median follow-up was $23.77 \pm 10$ months.

Results Twenty-one patients with IAAS were included. Indications to treatment were symptoms in 14 (67\%) patients and dilated abdominal aorta in $7(33 \%)$. Technical success was achieved in all cases. No perioperative mortality and 1 (4.8\%) early femoral access complication was encountered. During the follow-up were registered $1(4.8 \%)$ aortic unrelated death and $1(4.8 \%)$ stent-graft limb stenosis. The 36 months estimated survival and freedom from reintervention were 92\% (CI: 37-43; SE: 1.7 ) and 94\% (CI: 37-44; SE: 1.7), respectively.

Conclusions The endovascular treatment of IAAS with unibody stent-graft (AFX endovascular AAA system; Endologix Inc.) is safe and effective with promising mid-term outcomes. The use of unibody stent-grafts expands the endovascular indication, despite the usual anatomic IAAS features. Larger studies with longer follow-up are required to validate the outcomes of the reported technique.
\end{abstract}

Felice Pecoraro

felice.pecoraro@unipa.it

1 Department of Surgical, Oncological and Oral Sciences (Di.Chir.On.S.), University of Palermo, Via L. Giuffrè, 5, 90100 Palermo, Italy

2 Vascular Surgery Unit, AOUP "P. Giaccone”, Palermo, Italy

3 Vascular Surgery Unit, Ospedali Riuniti Villa Sofia-Cervello, Palermo, Italy

4 Aortic Center Hirslanden, Zurich, Switzerland

\section{Introduction}

The term "acute aortic syndrome" (AAS) is generally employed to describe life-threatening aortic diseases, that includes: intramural hematoma (IMH), penetrating aortic ulcer (PAU), and aortic dissection (AD) [1]. Despite different pathophysiology of such entities, a causal relationship has been proposed linking these three conditions, which represents a different stage of the same evolutive disease [2]. ADs represent the majority of AAS with a 
reported incidence of 2.6-3.5 cases per 100,000 personyears [3]. ADs are more frequently localized in the ascending aorta $(70 \%)$ followed by the descending aorta $(20 \%)$ and the aortic arch (7\%). Isolated abdominal aortic dissection (IAAD) is an extremely rare occurrence with a reported prevalence of $1.1-4 \%$ and in $91-96 \%$ present a spontaneous nature [4-6]. IMH accounts for approximately $6-10 \%$ of all AAS [7]. The true incidence of PAU has yet to be determined. However, abdominal PAU is less frequent than thoracic PAU with an estimated incidence of $1-5 \%[8]$.

As a result, the infrarenal localization of AAS is even rarer with limited and varied literature on the topic and unclear indications for medical, surgical, and endovascular treatment.

When an invasive IAAD treatment is indicated, the endovascular approach is privileged with fewer complications and mortality rates in the short and long term [5, 6]. Definitely no clear indication or evidence of superiority exists on the type of endovascular materials to employ.

A single-center experience of isolated infrarenal acute aortic syndrome (IAAS) treatment by endovascular means using unibody stent-graft is reported. The study aims to assess the feasibility of addressing IAAS lesions with a commercially available unibody stent-graft (AFX endovascular AAA system; Endologix Inc., Irvine, CA) and report the mid-term outcomes.

\section{Materials and methods}

From April 2016 to March 2019, data from consecutive patients presenting IAAS were prospectively collected, inserted into standardized piloted forms, and retrospectively analyzed. Patients treated with unibody stent-graft (AFX endovascular AAA system; Endologix Inc.) and presenting IAAS involving the infrarenal aorta were included in the study. AAS was defined according to the International Registry of Acute Aortic Dissection (IRAD) and included aortic dissection (AD), intramural hematoma (IMH), and penetrating aortic ulcer (PAU) [9]. Suprarenal AAS, post-traumatic AAS, or iatrogenic AAS was excluded. Patients managed with conservative therapy and open surgical treatments or addressed by endovascular means without the use of unibody aortic stent-grafts were also excluded from the current study. All patients gave the informed consent for the procedure itself, anonymous data collection, and analysis. According to the internal review board, the retrospective and anonymized nature of the study did not require medical ethical committee approval.

Indications to IAAS treatment were symptomatic IAAS and/or an associated dilated abdominal aorta $(>3 \mathrm{~cm})$.
Symptomatic IAAS were defined as abdominal/back pain, organ ischemia, distal embolization, and aortic rupture.

Demographic, comorbidities, and clinical data were collected. The preoperative types of imaging studies, procedure details, type of intervention, type of anesthesia, blood transfusions, medical therapy, and length of stay (LOS) were recorded.

Renal function was calculated according to the Chronic Kidney Disease Epidemiology Collaboration (CKD-EPI) formula [10]. Cardiac and respiratory function was assessed using The New York Heart Association (NYHA) heart function [11] and the Global Initiative for Chronic Obstructive Lung Disease (GOLD) [12].

The measured outcomes were technical success, early (within 30 days) and late (after 30 days) outcomes. Technical success was defined as procedure completion as intended with the complete exclusion of the IAAS and without unplanned additional maneuvers. Early outcomes included mortality, morbidity, symptoms recurrence, and endoleak occurrence. Late outcomes included mortality, symptoms recurrence, endoleak occurrence, stent-graft patency, and survival.

Follow-up consisted of clinical examination and duplex ultrasound (DUS) at 1 week; clinical examination and computed tomography angiography (CTA) at 1 and 12 months and yearly thereafter. Median follow-up was $23.77 \pm 10$ (mean: 22.53; interquartile range [IQR]: 15-28) months.

Statistical analysis. Parametric data are presented as mean and IQR or median and min-max range; absolute values and percentages for nonparametric data. Differences in preoperative and postoperative outcomes were assessed using the Student $t$ test. Kaplan-Meier curves were used to estimate survival. Statistical significance was considered at $p<.05$. For Kaplan-Meier curves, standard error exceeding $10 \%$ was reported. Statistical analysis was performed using SPSS 16.0 (SPSS Inc., Chicago, IL, USA).

\section{Results}

A total of 21 patients presenting IAAS and managed with the unibody stent-graft system (AFX endovascular AAA system; Endologix Inc.) were included in this study. During the same period, 15 patients presenting IAAS were observed and excluded from the current study. Three of them were managed by conventional open surgery and five by endovascular means without the unibody stent-graft system. Endovascular procedures were performed employing a Cheatham-Platinum (CP) stent (NuMED, Hopkinton, NY) in 2 patients, Viabahns (WL Gore and Associates, Flagstaff, AZ) in 1; and Endurant II stent-grafts (Medtronic Vascular, Santa Rosa, CA) in 2. Four morbid 
patients were left untreated and managed with the best medical therapy. The remaining three are under surveillance.

The mean age of included patients was $70.62 \pm 5$ (range 61-82) years, and 19 (91\%) were male. A previous heart and/or vascular intervention was reported in $13(62 \%)$ patients. Comorbidities are reported in Table 1. IAAS symptoms were recorded in $14(67 \%)$ patients, including abdominal/back pain in $10(48 \%)$ cases, distal embolization in $6(29 \%)$, and aortic rupture in $3(14 \%)$. Of these symptomatic patients, 1 presented with abdominal/back pain, distal embolization and aortic rupture; 2 with abdominal/back pain and aortic rupture; 1 with abdominal/ back pain and distal embolization; 6 with abdominal/back pain; and 4 with distal embolization. In the remaining 7 (33\%) cases, an abdominal aorta dilatation $>30 \mathrm{~mm}$ was the indication to treatment (Table 2).

The CTA scan was a diagnostic tool used to confirm the IAAS in all patients and to plan an operative strategy. In patients who had distal embolization, CTA was performed after clinical vascular evaluation and a duplex ultrasound

Table 1 Demographics and clinical preoperative data

\begin{tabular}{|c|c|}
\hline Number of patients & 21 \\
\hline Mean age, years & 70,62 \\
\hline Over 70 years, $n(\%)$ & $12(57)$ \\
\hline Female, $n(\%)$ & $2(9)$ \\
\hline Hypertension, $n(\%)$ & $19(91)$ \\
\hline Pulmonary disease, $n(\%)$ & $17(81)$ \\
\hline GOLD $1, n(\%)$ & 4 (19) \\
\hline GOLD $2, n(\%)$ & 4 (19) \\
\hline GOLD $3, n(\%)$ & $8(38)$ \\
\hline GOLD $4, n(\%)$ & $5(24)$ \\
\hline \multicolumn{2}{|l|}{ Cardiac disease, $n(\%)$} \\
\hline NYHA I, $n(\%)$ & $6(29)$ \\
\hline NYHA II, $n(\%)$ & $8(38)$ \\
\hline NYHA III, $n(\%)$ & 4 (19) \\
\hline NYHA IV, $n(\%)$ & $3(14)$ \\
\hline ASA III, $n(\%)$ & $14(67)$ \\
\hline ASA IV, $n(\%)$ & $7(33)$ \\
\hline Renal Function Impairment, $n(\%)$ & 4 (19) \\
\hline Mean GFR $\left(\mathrm{mL} / \mathrm{min} / 1.73 \mathrm{~m}^{2}\right)$ & 52 \\
\hline Dialysis & $2(9)$ \\
\hline Lipid disorder, $n(\%)$ & $12(57)$ \\
\hline Diabetes mellitus, $n(\%)$ & $6(29)$ \\
\hline Peripheral arterial disease, $n(\%)$ & $15(71)$ \\
\hline Cancer, $n(\%)$ & $7(33)$ \\
\hline
\end{tabular}

GOLD Global Initiative for Chronic Obstructive Lung Disease, $N Y H A$ New York Heart Association, ASA American Society of Anaesthesiologists, GFR glomerular filtration rate
Table 2 IAAS clinical presentation and anatomic features

\begin{tabular}{ll}
\hline Number of patients & 21 \\
Symptoms, $n(\%)$ & $14(67)$ \\
Abdominal/back pain, $n(\%)$ & $10(48)$ \\
Worse pain ever felt & 7 \\
Acute onset & 9 \\
$\quad$ Lacerating & 6 \\
$\quad$ Migratory & 4 \\
Distal embolization, $n(\%)$ & $6(29)$ \\
Aortic rupture, $n(\%)$ & $3(14)$ \\
Asymptomatic, $n(\%)$ & $7(33)$ \\
Aortic dissection, $n(\%)$ & $11(53)$ \\
Intramural hematoma, $n(\%)$ & $3(14)$ \\
Penetrating aortic ulcer, $n(\%)$ & $7(33)$ \\
Associated dilated abdominal aorta $(>3 \mathrm{~cm}), n(\%)$ & $11(53)$ \\
Iliac artery involvement, $n(\%)$ & $13(62)$ \\
\hline
\end{tabular}

of the lower limbs. For asymptomatic patients, 5 were already inserted into a follow-up protocol; the remaining 2 were incidentally diagnosed.

An $\mathrm{AD}$ was recorded in $11(53 \%)$ cases, an IMH in 3 $(14 \%)$ and a PAU in $7(33 \%)$. An associated dilated (>30 mm) abdominal aorta was detected in $11(53 \%)$ patients (4 symptomatic and 7 asymptomatic); and a dissection of the iliac artery in $13(62 \%)$ (Table 2). In $4(19 \%)$ patients, the distance from the lowest renal artery to the aortic bifurcation was less than $80 \mathrm{~mm}$; in $7(33 \%)$ patients, the mean aortic diameter at iliac bifurcation was less than $16 \mathrm{~mm}$; the combination of both anatomic findings was observed in $4(19 \%)$. The main imaging findings are reported in Table 3. In the present study, the unibody stent-graft system (AFX endovascular AAA system; Endologix Inc.) was introduced and deployed according to the standard technique for abdominal aortic aneurysm repair (Fig. 1). In 13 (62\%) cases, a proximal extension was planned. In 9, the proximal extension was undertaken using a VELA infrarenal cuff (Endologix, Inc.); in 2 a covered CP stent (NuMED Inc.); and in the remaining 2 an Endurant II (Medtronic) proximal extension (1 aortic tube and 1 aortic cuff) (Fig. 2). A proximal extension was decided at operator discretion according to the anatomic findings of the proximal neck. Aortic body and proximal extension features are reported in Table 4.

In $10(48 \%)$ patients, a post-dilation of the unibody stent-graft iliac limbs was planned. In 3 patients, the dilation was performed by kissing balloon of the aortic bifurcation.

Interventions were carried under local anesthesia in 19 (90\%) patients; general anesthesia in $1(5 \%)$; and spinal anesthesia in $1(5 \%)$. A totally percutaneous approach was 
Table 3 IAAS imaging findings

\begin{tabular}{|c|c|}
\hline & Mean (IQR) \\
\hline Lowest renal artery to aortic bifurcation distance, $\mathrm{mm}$ & $88.2(81-95)$ \\
\hline Aortic bifurcation diameter, $\mathrm{mm}$ & $18.5(16-20)$ \\
\hline Maximal aortic diameter, $\mathrm{mm}$ & $29.3(20-42)$ \\
\hline Right iliac artery distal landing zone diameter, $\mathrm{mm}$ & $11.1(9-13)$ \\
\hline Left iliac artery distal landing zone diameter, $\mathrm{mm}$ & $10.9(9-12)$ \\
\hline Proximal infrarenal neck angulation, ${ }^{\circ}$ & $16.3(11-23)$ \\
\hline Lowest renal artery to primary entry site distance, $\mathrm{mm}$ & $28.4(25-33)$ \\
\hline Proximal neck diameter al lowest renal artery & $20.9(18-24)$ \\
\hline
\end{tabular}

$I Q R$ interquartile range

employed in $17(81 \%)$ patients according to the standard technique [13]. The mean intervention duration was $108 \pm 43$ IQR: 75-135) minutes. In the $11(52 \%) \mathrm{AD}$ cases, the intravascular ultrasonography (IVUS) was employed.

During the hospital stay, all patients received prophylactic low molecular weight heparin (LMWH) and single antiplatelet therapy (100 mg acetylsalicylic acid). After discharge, the single antiplatelet was continued. For patients reporting preoperative anticoagulation, a switch to therapeutic LMWH before the intervention was performed. The medical management included B-blockers preferentially administered to control blood pressure and statins for lipid disorder.

Technical success was achieved in all cases. No perioperative mortality was registered. In $1(4.8 \%)$ patient, a common femoral artery occlusion requiring a patch angioplasty and distal embolectomy on 2nd POD occurred. No symptomatic recurrence or early endoleaks were detected. During the follow-up, 1 (4.8\%) patient died after 21 months of cancer. At 14 months of follow-up, 1 patient required balloon-expandable stent placement for right iliac limb stenosis and claudication. No endoleak or aortic stentgraft occlusion were recorded. At 36 months, the estimated survival and freedom from reintervention were $92 \%$ (CI: 37-43; SE: 1.7) and 94\% (CI: 37-44; SE: 1.7), respectively (Fig. 3).

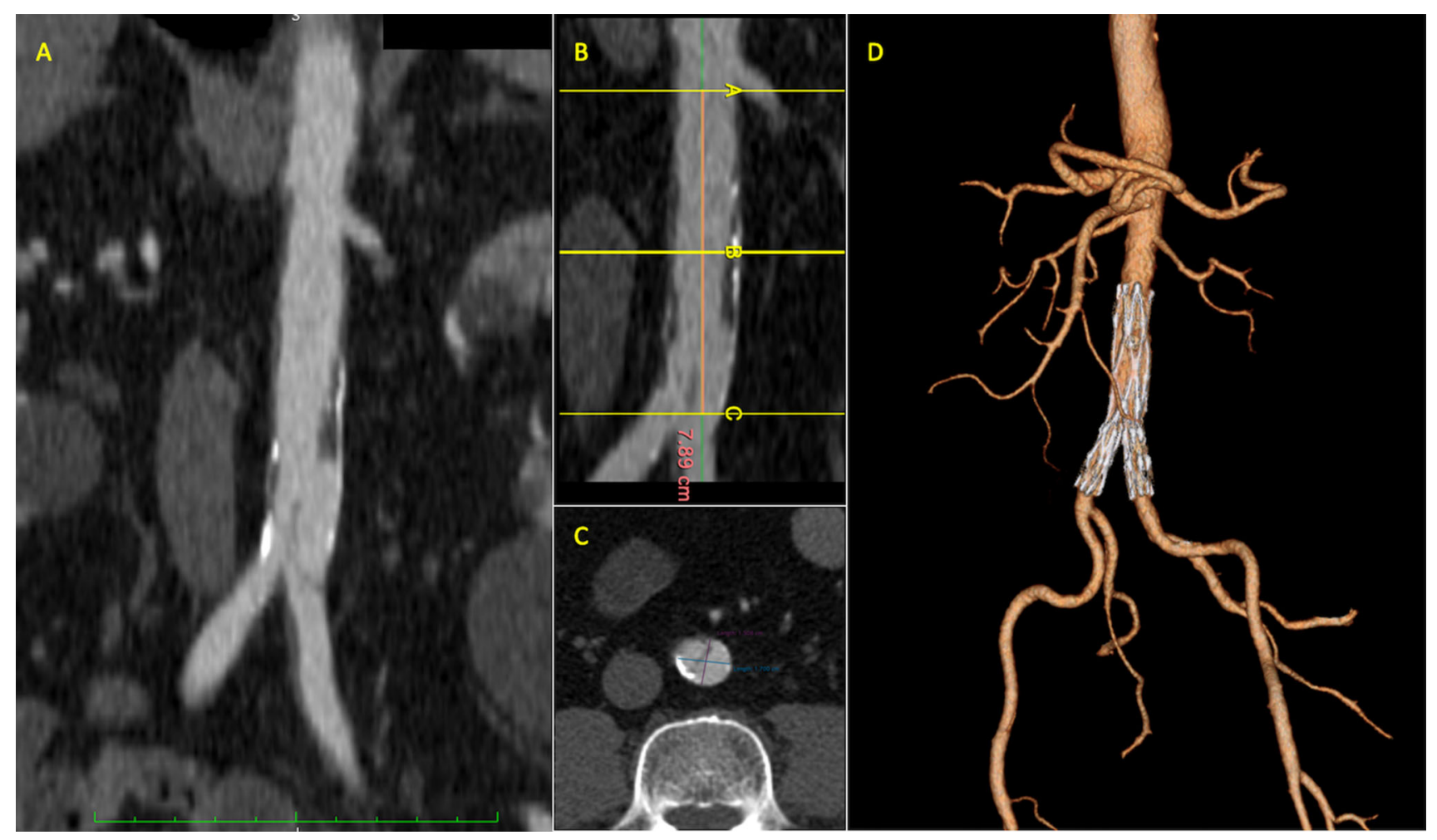

Fig. 1 Preoperative CTA with maximum intensity projection (MIP) showing an infrarenal aortic dissection, involving of the origin of the left common iliac artery (a); centerline showing a limited length of the infrarenal aorta (less than $8 \mathrm{~cm})(\mathbf{b})$; axial projection showing the dissection flap in correspondence of the distal abdominal aorta with limited aortic diameter (approx. $17 \times 15 \mathrm{~mm}$ ) (c); postoperative 24-month follow-up CTA 3D reconstruction showing the exclusion of dissection with patent true lumen and disappearance of the false lumen (d) 


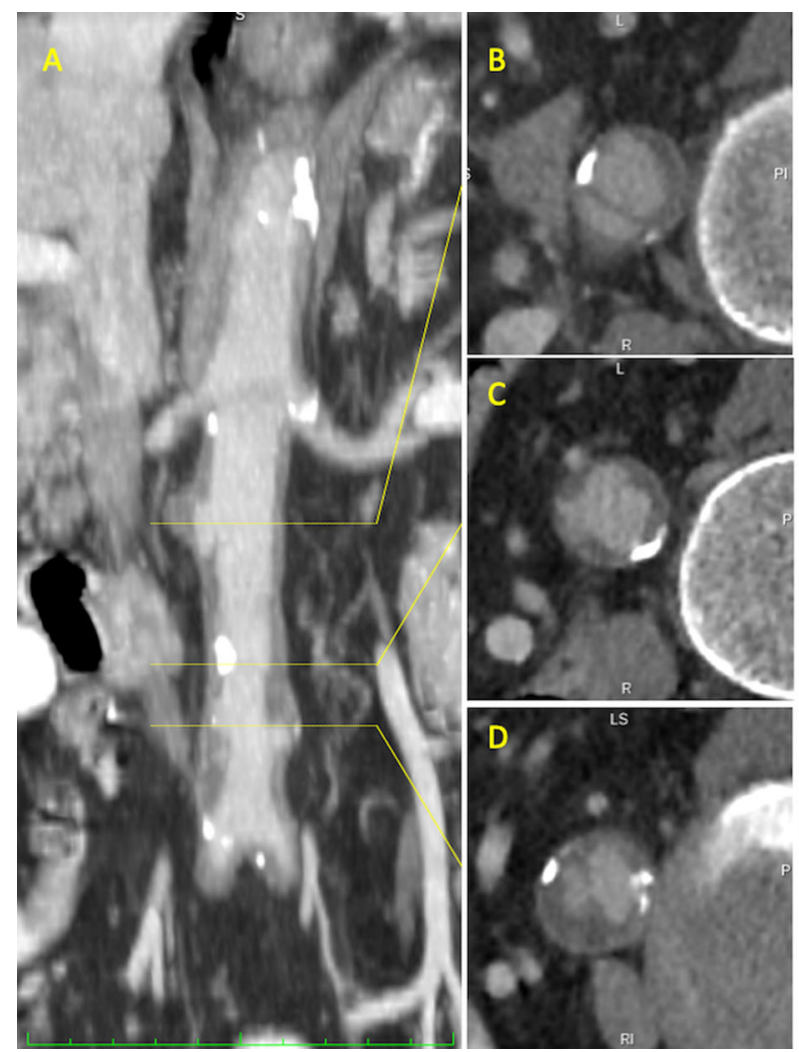

Fig. 2 Preoperative CTA MIP showing overall infrarenal acute aortic syndrome (a). Infrarenal aortic dissection (b), intramural hematoma (c) and penetrating aortic ulcer (d), intraoperative arteriogram after

Table 4 Aortic body and proximal extension features

\begin{tabular}{ll}
\hline & Mean (IQR) \\
\hline Aortic body length, mm & $78.6(70-80)$ \\
Aortic body diameter, mm & $24.1(22-25)$ \\
Aortic body iliac length, mm & $36.3(30-40)$ \\
Aortic body iliac diameter, mm & $16.2(16-16)$ \\
Proximal cuff length, mm & $76.1(75-80)$ \\
Proximal cuff diameter, mm & $27.1(25-28)$ \\
\hline
\end{tabular}

\section{Discussion}

AAS is generally reported in the thoracic aorta; among all AAS, the prevalence of classic AD is the highest over IMH and PAU, respectively, with a prevalence of $85-95 \%$, 5-27\%, and 2-7\% [14, 15].

Current literature lacks incidence data on AAS of the abdominal aortic segment. Most of the publications address IAAD, PAU, or IMHs issues separately and the majority of them are on IAAD [5, 6]. A recent systematic review estimated an IAAD incidence of 5,1 per 1,000,000 persons

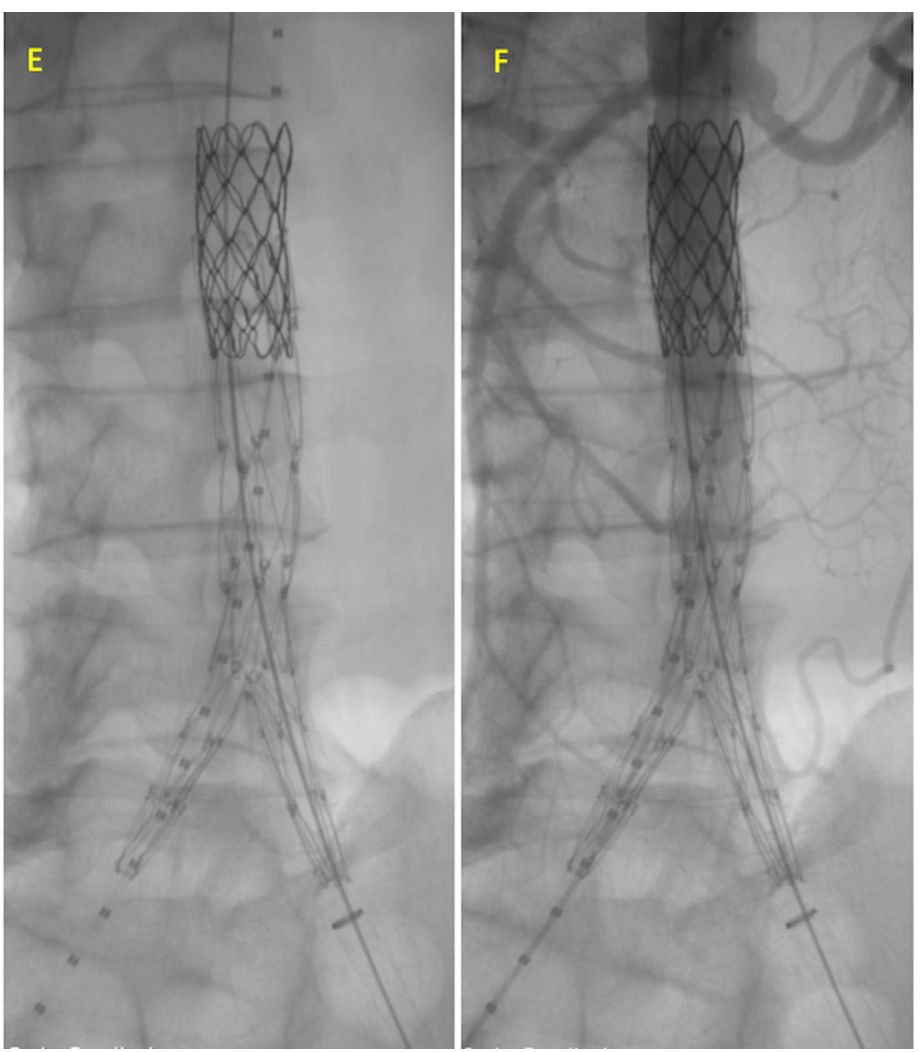

unibody stent-graft deployment and proximal extension (e), and after contrast media injection (f)

annually, and an infrarenal aortic segment localization in $82 \%$ [5].

Overall, the literature reports up to 500 cases of IAAD and 300 cases of abdominal PAU [5, 6, 16].

Somehow in line with our study, we had an IAAD incidence of 53\%, abdominal PAU 33\%, and abdominal IMH $14 \%$. From it, a speculative incidence of IAAS could be reported of 10 persons per 1,000,000 annually.

Anyway, IAAS is usually reported in males over 60 years and can be secondary to trauma, iatrogenic, or spontaneous [17]. The natural history of IAAS is a rupture in up to $15 \%$ of patients and a mortality rate ranging from 17 to $28 \%$ [18].

As recommended by Farber et al., a 3-cm abdominal aortic diameter should be considered for elective treatment due to the higher rupture risk compared to abdominal aortic aneurysm (AAA) and the unknown natural history of aneurysmal degeneration of the dissected abdominal aorta [19].

IAAS can be managed with the medical, surgical, or endovascular approach. An insight from the IRAD reports that the medical management on 18 patients presenting an IAAS was significantly associated with a higher mortality 
Fig. 3 Survival and freedom from reintervention estimated 3-year Kaplan-Meier curves. Standard error does not exceed $10 \%$ at 3 years for both survival curves

risk when compared to operative treatments, suggesting an aggressive surgical or endovascular repair [9].

The endovascular approach showed better results with a reduced mortality and morbidity risk when compared to conventional open surgery $[5,6,17]$.

The main concerns of IAAS endovascular treatments are the lack of dedicated devices and the use of standard aortic stent-grafts outside the instruction for use (IFU). Different solutions including uncovered stents, covered stents, and stent-grafts have been proposed [18].

Due to the risk of distal embolization, the use of uncovered stents is limited to patients presenting IAAS without thrombus. Covered stents and stent-grafts are employed in most patients; moreover, the use of stent-graft is mandatory in case of associated infrarenal aortic aneurysm [20].

IAAS management with different types of aortic stentgraft has been associated with good results, even in terms of aortic remodeling and false lumen thrombosis; however, limb occlusions and persistent endoleaks are the main cause of reinterventions [5, 6, 21].

IAAS generally presents specific anatomic features including a short/limited length of the infrarenal aorta, and a narrow arterial lumen, especially at aortic bifurcation and iliac arteries. The association with aneurysmal dilation generally worse the IAAS anatomy; in these circumstances, the aortic true lumen is further narrowed by the false lumen from which the aneurysmal dilatation generally arises [22].
To overcome these anatomic limitations, different endovascular solutions including the use of aortic tube, aortic kissing stent-grafting, or standard aortic stent-graft have been reported. The use of aortic tube can be indicated in IAAS with no iliac involvement with a potential risk of migration due to the lack of distal iliac fixation. The limitations of the kissing stent-graft technique are related to the shape changes of the abdominal aorta, the risk of proximal misaligning, and the risk of occlusion.

The usual IAAS anatomic features represent major limitations also to the available standard bi/trimodular aortic stent-grafts. In particular, the short distance from the lowest renal artery and the aortic bifurcation has the risk of contralateral gate opening inside the homolateral iliac introduction site; the narrowed diameter in correspondence of the aortic bifurcation has the risk of contralateral gate incomplete opening or collapse inside the distal aorta. Aorto-uni-iliac stent-grafts represent another option but require an additional femoro-femoral bypass.

A recent publication reported IAAD treatment with Aegis $^{\text {TM }}$-B unibody stent-graft (Microport, Shanghai, China) in 32 patients, with good results, no events of mortality within 90 days and 1 limb occlusion [23]. Moreover, they reported the use of proximal cuff extension only in $3 / 32(9.3 \%)$ case, in contrast with our report of $13 / 21(62 \%)$, most probably due to the shorter aortic length of Asiatic population. However, Aegis ${ }^{\mathrm{TM}}$-B (Microport) is not available on the EU and US markets. 
In our experience, the best commercially available device adapting to IAAS characteristic was the unibody stent-graft system (AFX endovascular AAA system; Endologix Inc.). The bottom-up construction of such a device allows its use in small aortic bifurcation diameter and short renal to aortic bifurcation distance. Further, the unibody stent-graft system (AFX endovascular AAA system; Endologix Inc.) avoid the gate cannulation issues related to conventional bi/trimodular stent-grafts in such a small aortic diameter (mean $29.3 \mathrm{~mm}$ ) as reported in our cohort.

These anatomic issues are uncommon for AAA intended for treatment $(>5-5.5 \mathrm{~cm})$, exploiting greater infrarenal aortic length and intra-aortic space for device maneuvers. In fact, the AFX endovascular AAA system (Endologix Inc.) device is designed to treat AAAs and IFU does not include IAAS. The recent FDA concerns about type III endoleaks have prompted the company to review and issue security updates for AFX (Endologix Inc.) devices. The investigations into type III endoleaks identified several contributing factors, including an inadequate component overlap and a large or tortuous aorta: on these bases, Endologix introduced an IFU update on sizing algorithm for the AFX (Endologix Inc.) platform to maximize overlap, calculated on the aneurysm radius $+20 \mathrm{~mm}$.

Obviously, the stated indications regarding the endoleak issues are irrelevant in the case of IAAS treatment. Theoretically, IAAS follow-up should be uneventful for type III endoleaks and further investigation in regard could clarify the role of the AFX endovascular AAA system (Endologix Inc.) device.

In this series, $15(71 \%)$ patients exhibited at least one anatomic feature not fitting for conventional bi/trimodular bifurcated graft. Based on this experience, the IAAS anatomic limitations to the use of the unibody stent-graft system (AFX; Endologix Inc.) are principally related to the distance from the aortic bifurcation to the origin of the internal iliac arteries (IIAs), as $a<30 \mathrm{~mm}$ length pones the risk of IIA occlusion. Restricted aortic bifurcations $(<14 \mathrm{~mm})$ also represent an increased risk of guiding each limb into its respective common iliac artery.

IVUS is an additional endovascular tool to identify the entry tear of $\mathrm{AD}$, vessel diameters and lengths [24, 25]. In our practice, this device was useful to confirm guidewire intraluminal route and it was employed in all patients (52\%) presenting with IAAD.

Limitations of the present study are the retrospective analysis and the limited number of included patients. The major limitation is the absence of a comparative groups treated by open surgery or other endovascular means. In addition, a relevant bias is the lack of randomization of the approach chosen, including open surgery/unibody stentgraft/trimodular stent-graft/covered stent-grafts. Also, the follow-up duration is a limitation to drawing robust conclusions.

\section{Conclusions}

In the reported experience, the endovascular treatment of IAAS was safe and effective with promising mid-term outcomes. The use of unibody stent-grafts expands the endovascular indication despite the usual anatomic IAAS features. Larger studies with longer follow-up are required to validate the outcomes of the reported technique.

Funding Open access funding provided by Universit Ã degli Studi di Palermo within the CRUI-CARE Agreement.

\section{Compliance with ethical standards}

Conflict of interest The authors declare that they have no conflict of interest.

Ethical standards All procedures followed were in accordance with the ethical standards of the institutional committee on human experimentation and with the Helsinki Declaration.

Informed consent Informed consent was obtained from all individual participants included in the study.

Open Access This article is licensed under a Creative Commons Attribution 4.0 International License, which permits use, sharing, adaptation, distribution and reproduction in any medium or format, as long as you give appropriate credit to the original author(s) and the source, provide a link to the Creative Commons licence, and indicate if changes were made. The images or other third party material in this article are included in the article's Creative Commons licence, unless indicated otherwise in a credit line to the material. If material is not included in the article's Creative Commons licence and your intended use is not permitted by statutory regulation or exceeds the permitted use, you will need to obtain permission directly from the copyright holder. To view a copy of this licence, visit http://creativecommons. org/licenses/by/4.0/.

\section{References}

1. Vilacosta I, San Román JA (2001) Acute aortic syndrome. Heart Br Card Soc 85:365-368. https://doi.org/10.1136/heart.85.4.365

2. Pecoraro F, Rancic Z, Mayer D et al (2011) Acute aortic dissection: a case report showing penetrating thoracic aortic ulcer and infrarenal aortic rupture. J Cardiovasc Med Hagerstown Md 12:655-656. https://doi.org/10.2459/JCM.0b013e328348e560

3. Corvera JS (2016) Acute aortic syndrome. Ann Cardiothorac Surg 5:188-193. https://doi.org/10.21037/acs.2016.04.05

4. Roberts CS, Roberts WC (1991) Aortic dissection with the entrance tear in abdominal aorta. Am Heart J 121:1834-1835

5. Wu J, Zafar M, Qiu J et al (2019) A systematic review and metaanalysis of isolated abdominal aortic dissection. J Vasc Surg 70:2046-2053.e6. https://doi.org/10.1016/j.jvs.2019.04.467 
6. Liu Y, Han M, Zhao J et al (2020) Systematic review and metaanalysis of current literature on isolated abdominal aortic dissection. Eur J Vasc Endovasc Surg Off J Eur Soc Vasc Surg 59:545-556. https://doi.org/10.1016/j.ejvs.2019.05.013

7. Eggebrecht H, Plicht B, Kahlert P, Erbel R (2009) Intramural hematoma and penetrating ulcers: indications to endovascular treatment. Eur J Vasc Endovasc Surg Off J Eur Soc Vasc Surg 38:659-665. https://doi.org/10.1016/j.ejvs.2009.09.001

8. Batt M, Haudebourg P, Planchard P-F et al (2005) Penetrating atherosclerotic ulcers of the infrarenal aorta: life-threatening lesions. Eur J Vasc Endovasc Surg Off J Eur Soc Vasc Surg 29:35-42. https://doi.org/10.1016/j.ejvs.2004.09.025

9. Trimarchi S, Tsai T, Eagle KA et al (2007) Acute abdominal aortic dissection: insight from the International Registry of Acute Aortic Dissection (IRAD). J Vasc Surg 46:913-919. https://doi. org/10.1016/j.jvs.2007.07.030

10. Levey AS, Stevens LA, Schmid CH et al (2009) A new equation to estimate glomerular filtration rate. Ann Intern Med 150:604-612. https://doi.org/10.7326/0003-4819-150-9200905050-00006

11. Dolgin M, Committee of the New York Heart Association (1994) Nomenclature of diseases of the heart and great vessels. Little Brown \& Co, Boston

12. From the global strategy for the diagnosis, management and prevention of copd, global initiative for chronic obstructive lung disease (gold) 2019. http://goldcopd.Org/. Accessed 10 Sept 2019

13. Pecoraro F, Krishnaswamy M, Steuer J et al (2017) Predilation technique with balloon angioplasty to facilitate percutaneous groin access of large size sheath through scar tissue. Vascular 25:396-401. https://doi.org/10.1177/1708538116688786

14. Bossone E, LaBounty TM, Eagle KA (2018) Acute aortic syndromes: diagnosis and management, an update. Eur Heart $\mathbf{J}$ 39:739-749d. https://doi.org/10.1093/eurheartj/ehx319

15. Evangelista A, Maldonado G, Moral S et al (2019) Intramural hematoma and penetrating ulcer in the descending aorta: differences and similarities. Ann Cardiothorac Surg 8:456-470. https:// doi.org/10.21037/acs.2019.07.05

16. Kotsis T, Spyropoulos BG, Asaloumidis N et al (2019) Penetrating atherosclerotic ulcers of the abdominal aorta: a case report and review of the literature. Vasc Spec Int 35:152-159. https:// doi.org/10.5758/vsi.2019.35.3.152
17. Jonker FHW, Schlösser FJV, Moll FL, Muhs BE (2009) Dissection of the abdominal aorta. current evidence and implications for treatment strategies: a review and meta-analysis of 92 patients. J Endovasc Ther 16:71-80. https://doi.org/10.1583/082601.1

18. Giribono AM, Ferrara D, Spalla F et al (2016) Endovascular treatment of spontaneous isolated abdominal aortic dissection. Acta Radiol Open 5:205846011668104. https://doi.org/10. 1177/2058460116681042

19. Farber A, Wagner WH, Cossman DV et al (2002) Isolated dissection of the abdominal aorta: clinical presentation and therapeutic options. J Vasc Surg 36:205-210. https://doi.org/10.1067/ mva.2002.125028

20. Jawadi N, Bisdas T, Torsello G et al (2014) Endovascular treatment of isolated abdominal aortic dissections: long-term results. J Endovasc Ther Off J Int Soc Endovasc Spec 21:324-328. https://doi.org/10.1583/13-4467MR.1

21. Zhu Q, Li D, Lai M et al (2015) Endovascular treatment of isolated abdominal aortic dissection and postoperative aortic remodeling. J Vasc Surg 61:1424-1431. https://doi.org/10.1016/j. jvs.2015.01.027

22. Adam DJ, Roy-Choudhury S, Bradbury AW (2007) Endovascular repair of spontaneous infrarenal aortic dissection presenting as severe lower extremity ischaemia. Eur J Vasc Endovasc Surg Off J Eur Soc Vasc Surg 34:699-701. https://doi.org/10.1016/j.ejvs. 2007.05.018

23. Zhou M, Cai H, Li Z et al (2018) Contemporary results of endovascular repair of isolated abdominal aortic dissection with unibody bifurcated stent grafts. Ann Vasc Surg 49:99-106. https://doi.org/10.1016/j.avsg.2017.10.035

24. Giudice R, Frezzotti A, Scoccianti M (2002) Intravascular ultrasound-guided stenting for chronic abdominal aortic dissection. J Endovasc Ther 9:926-931. https://doi.org/10.1177/ 152660280200900631

25. Pecoraro F, Bracale UM, Farina A et al (2019) Single-center experience and preliminary results of intravascular ultrasound in endovascular aneurysm repair. Ann Vasc Surg 56:209-215. https://doi.org/10.1016/j.avsg.2018.09.016

Publisher's Note Springer Nature remains neutral with regard to jurisdictional claims in published maps and institutional affiliations. 